\author{
Krzysztof Powała \\ Department of Environmental Engineering, Faculty of Process and Environmental Engineering, Lodz \\ University of Technology \\ Poland, krzysztof.powala@edu.p.lodz.pl
}

THE INFLUENCE OF SELECTED POLYMERS ON CONCRETE TIGHTNESS - CURRENT STATE OF KNOWLEDGE

\begin{abstract}
Today's construction market of materials, contractors and building users, places new demands on individual building materials. Undoubtedly one such material is concrete, which is used in almost all construction projects as a binder for building materials. Therefore, it is necessary that this material is constantly subjected to new tests to find ways to improve its properties. Therefore, many scientists attempt to improve properties such as compressive strength, flexural strength and water resistance. One method is to modify the cement matrix with polymers.
\end{abstract}

Keywords

cement, leakage, phase change material, waterproof

\title{
Introduction
}

Polymers were already in use in the 1980s, however, there were problems not only with the possibility of mixing polymers with cement as well as the costs of production. The idea was temporarily abandoned but resurfaced about 25 years later. The idea of using polymers in cement has begun to show reliable results and with continued progress and decreasing production costs may cause a major revolution in the building materials market in the near future. Based on today's research, scientists are obtaining good results in improving cement's flexural strength, compressive strength, water tightness and resistance to low temperatures.[1] Another important aspect is the thermal insulation obtained by adding polymer to mortar. For this purpose, phase-change materials such as paraffin are often used. Regarding measurements for the use of polymers and then paraffin, it is reported in the literature, that the water-tightness of the cement is important. Polymers added to cement allows to achieve a layer that is watertight to some extent. When using paraffin, the concept of adding polymer is similar except that the polymer admixture creates a layer to prevent cement paraffin from leaking. It can be assumed that if a good quality polymer is used, it will create a suitable impermeable layer. Such a layer will allow the paraffin to remain inside the material if the state of aggregation changes.[2] Cement modified in this way will obtain good insulating properties. This work is a literature review, the results of research on the various types of polymers is presented below.

\section{Types of reinforced cement}

On the basis of over a dozen years of research on the application of polymers in cement, we managed to draw some interesting conclusions. First, it was possible to group polymers based on their use in cement. Therefore, we can distinguish three groups: polymer impregnated concrete (PIC), polymer concrete (PC) and polymer modified concrete (PMC). "Polymer impregnated concrete was first produced in the 1960s. PIC is made by impregnating the cement with a low viscosity monomer which is them later polymerized. The advantage of this solution is increased tensile and bending strength. Also, this solution increases durability to weather conditions. Thanks to such properties PIC was eagerly used in places of joining the structure, as a support for pipes, as well as in places with high stresses" [3]. Concrete polymers were already in use in 1958, which included aggregate as well as a polymer binder. Interestingly, this cement does not contain water. Polystyrene as well as various types of acrylics and epoxies were the most commonly used monomers. Various types of resins were also used, as well as sulfur, which is considered to be a polymer. Concrete with the addition of sulfur is used primarily in places where resistance to acidic compounds is required. Such material is characterized by high durability, which is why it was often used as a repair material. Unfortunately, PC is characterized by high thermal expansion which can cause the part being repaired to fragment. Unfortunately, PC has been supplanted by Portland concrete, which is cheaper to produce as well as cures faster. Polymer concrete is mainly used for the construction of tanks, drains, curbs as well as floor tiles. 
The third type of concrete is polymer-modified concrete. It consists of Portland cement and modified cement with polymers such as acrylic, SBR and others. The polymers in PMC amount to only $20 \%$, however, oftentimes it gives poor results. This type of cement is characterized by high strength as well as low permeability. Thanks to this type of cement, compression can be increased, thus reducing the chances of cracking. With this technology, the consumption of polymer is smaller, which means it is cheaper to produce than PC.

\section{Requirements and selection of polymers (General concept of using polymers)}

Research based on mixing polymer together with cement is primarily carried out to find ways to improve their properties. This is important due to the nature of the facilities where improved cement will be used, which is mainly construction projects such as bridges and overpasses where the cement will be exposed to high stresses. Therefore, the added polymers must meet several important conditions so that the intended structure remains intact as long as possible.

One important condition is the thermal stability of the polymer. Due to the prevailing temperatures in summer and winter, it is important that the polymer used is thermally stable. During higher temperatures followed by cold, irreversible effects can occur and cause deformations in the material. This is an undesirable feature that eliminates the use of polymer. It is estimated that polymer has a greater tendency to expand than metal.

Polymers are a good insulating material characterized by low thermal conductivity which varies from 0.15 $\left[\mathrm{W} / \mathrm{m}^{*} \mathrm{~K}\right]$ to $0.3\left[\mathrm{~W} / \mathrm{m}^{*} \mathrm{~K}\right]$ and in some cases may be even lower. Therefore, polymers may play an additional insulating role.

Another issue is the resistance of the polymer to weather conditions. Based on local weather conditions an appropriate polymer, or additional substances that will stabilize the polymer can be selected. As mentioned previously, the temperature affects the polymer's expansion. However, in addition to the surface temperature, polymer can also be affected by ultraviolet rays, high energy radiation and the chemical environment in the form of acid rain. Each polymer will react differently to external factors, for example, causing discoloration of a wall as well as cracking or destruction of the wall from the inside. Depending on the materials used It is important to properly preserve external surfaces, however, the aging process cannot be entirely eliminated.

Another important feature of the polymer is the degree of permeability through the material layer. Polymers are used successfully as sealing agents in various forms for both vertical and horizontal surfaces. This will be described in more detail in a later part of the text

Another feature of polymers is their flammability. Unfortunately, any organic or synthetic polymer is flammable. Depending on the composition, polymers give off toxic substances that threaten human health. However, both synthetic and natural polymers are used in construction within a certain range of fire safety. In non-combustible conditions, polymers can produce adverse effects to the properties of the surface. Therefore, when using materials such as polymers that are located in the fire risk zone of the buildings concerned, an appropriate sign designating the degree of fire hazard should be posted.

The greatest enemy of cement is the corrosion of reinforcement bars caused by continuous temperature changes. Research has determined that the durability of concrete depends on its porosity. The less porous concrete is, the stronger it becomes. The porosity affects the penetration of water, therefore admixtures of plasticized blends have been proposed. Due to the addition of such substances, the durability of cement is clearly increased.

To reduce the penetration of water, various types of additives are used. They are divided into normal water reducers and large range reducers. The first group are derived from waste during the production of pulp and can reduce the share of water to $10 \%$. The second group is admixtures in the form of polymers. The use of reducers with a large range can reduce the use of water up to $40 \%$. The use of this type of substance not only causes higher durability but also allows the use of such reinforced cement in inaccessible places by increasing the feasibility of application.

The influence of water on cement functions in the following way. When cement particles are dry, a small amount of water will cause electrical charges to merge, which in this case, causes the water to dissipate incorrectly for the cement. The use of polymers prevents cement particles from being combined, which after some time can be 
isolated as a small deposit. The operation is based on combining particles that can have both a positive and a negative charge which, in contact with other molecules, leads to flocs. The polymers used in such a mixture act as dispersants, i.e. they prevent particles from combining by giving the particles negative charges, which leads to the repulsion of particles. A kind of shell is formed around the cement particle, pushing other particles further away. Thanks to this action, the water molecules cannot combine cement particles into larger agglomerates. After the polymer is added, the fresh concrete is in an easy to apply liquid form, which preserves all properties of concrete without strengthening after solidification.

When characterizing concrete, the workability of the concrete mix should be mentioned. Workability is referred to as the cement's ability to spread easily in a given place. In short, workability indicates if the cement is in a sufficient enough liquid enough or not. Cement is often brought to a construction site in liquid form and it is important that it remains in this form. In some cases, the workability becomes reduced and some water is added to increase workability. To avoid such situations, the polymer is added to the concrete on site or more appropriately at the beginning of mixing to prevent aggregation of the cement. However, this can be economically costly, especially the addition of large doses of polymer.

During the process of concrete curing, it is important that the mixed and prepared cement cures as much as possible in the most saturated environment possible. If too little water is added to the mix it may cause selfdrying of the concrete. This is a dangerous phenomenon that can lead to undesired shrinkage of the concrete and, consequently, to cracks. Such concrete does not have high strength properties nor is it resistant to weather conditions. In some cases, appropriate self-hardeners are added to avoid this problem taking place. Selfhardeners usually take up absorbent polymers. They prevent cement from cracking too quickly, reduce permeability and increase durability. The principle of operation of such polymers is quite simple as they can absorb a large amount of water because such polymers have an ionic nature.

\section{Using polymers in concrete - mechanical properties}

The literature review shows the results of the use of several polymers as an example for further studies on their effect on waterproofness of cement. One of the best polymers, which is styriene-butiadene rubber, has been considered. One of the most important advantages of this polymer is its ability to create a surface containing a smaller amount of pores than in the case of unmodified cement. This is important because in many cases it is the polymer that fills the pores thus ensuring waterproofness even with a low content of up to $2 \%$.

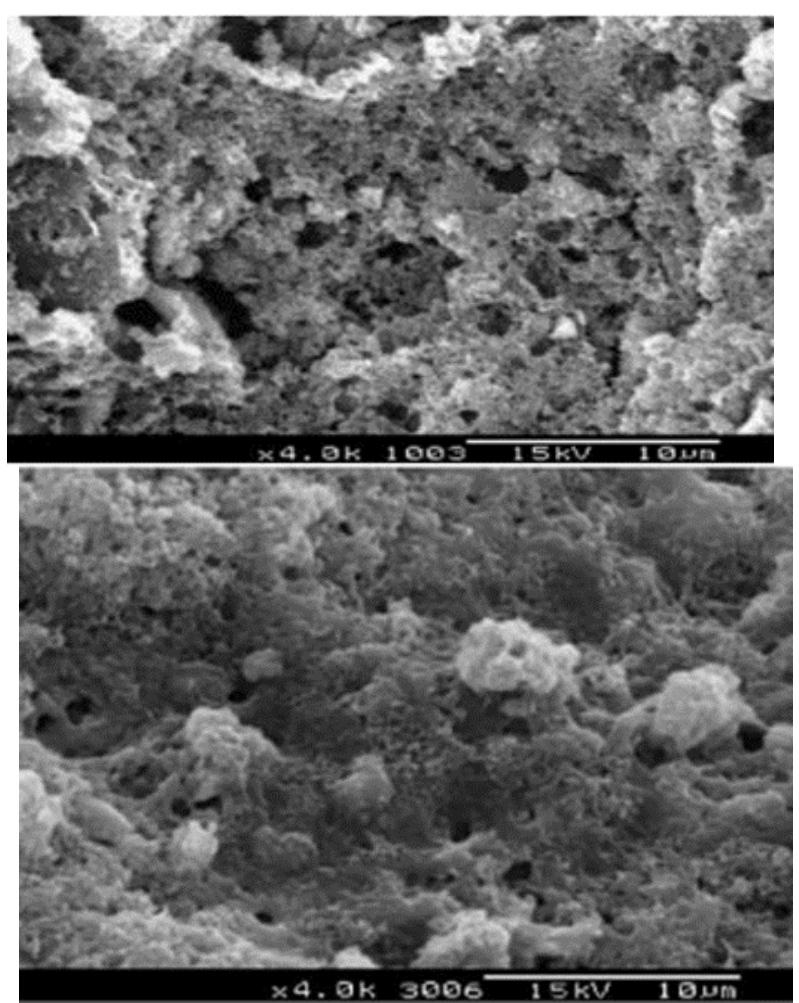

Fig. 1 Cement structure with 0\% (top) and 5\% polymer (bottom) (SBS) [4] 
In Figure 1, the cement structure using SBS in the amount of 5\% is not uniform but the surface is much more sealed than in the case of non-upgraded cement. As a result, not only water-tightness but also other properties are significantly improved. The highest density is obtained at $2 \%$ of the SBS content, after which it decreases and then increases once again at a content level of $8 \%$. The compression strength is similar, the highest value is achieved at $2 \%$ content, then it decreases and again increases at $10 \%$. A polymer concentration of approx. $2 \%$ allows for the highest flexural strength. The polymer has better retention properties, which increases the porosity of the mortar. As can be seen, SBS is one of many examples that improves the properties of cement. Figs. 2 and 3 show the change in compressive and flexural strength depending on the amount of polymer in the cement.

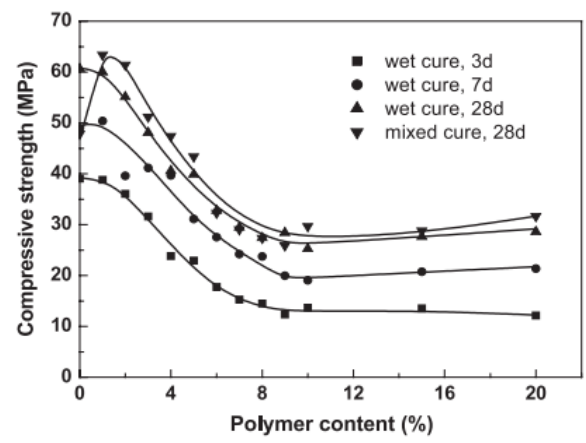

Fig.2 Compressive strength of polymer-modified mortars with different polymer/cement mass ratios Source : [4]

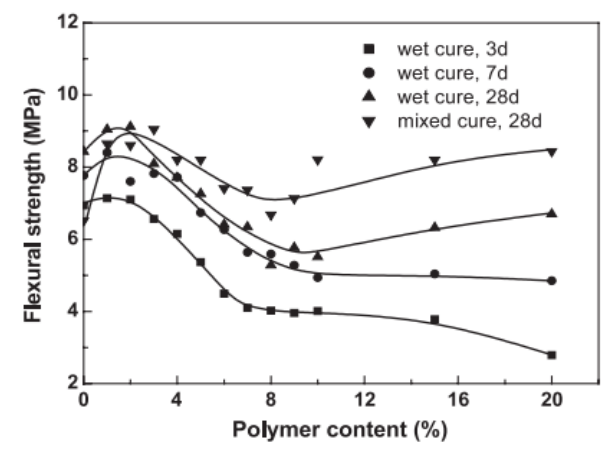

Fig.3 Flexural strength of polymer modified mortars with different polymer/cement mass ratios Source: [4]

Other interesting polymers that can be successfully used in cement are styrene-acrylic ester and ethylene-vinyl acetate. Comparing them, we can draw the following conclusions. Styrene-butiadiene rubber improves flexural strength, tensile strength and water resistance. Styrene-acrylic ester reduces the modulus of elasticity and increases the strength of the mortar. Ethylene-vinyl acetate improves flexural strength and stretching [5]. The value of water adsorption decreases to the largest extent below $7 \%$. However, when the polymer content is more than $7 \%$ in the cement, it is approx. $0.7 \mathrm{~kg}^{*} \mathrm{~m}^{-2}$ and is better than ethylene-vinyl where the content is $0.8 \mathrm{~kg}^{*} \mathrm{~m}^{-2}$. However, it is not as good as SBR, where the value is $5 \%$ and is $0.7 \mathrm{~kg}^{*} \mathrm{~m}^{-2}$.

\section{Water impermeability}

The most important parameter that will allow the use of polymer in cement is its water impermeability. As mentioned before, this is the basis for extending the durability of cement. The main emphasis should be on cement reinforced with rods because corrosion can occur in such cement. This leads to many undesirable effects, mainly cracks. Such damaged cement quickly loses its properties, which is unacceptable in the case of structures that carry heavy loads. Therefore, scientists aim to solve the problem of water resistance. In concrete that is not reinforced with polymer, water penetrates the concrete deeply, which causes dampness to persist for a long time due to the high porosity of the cement. Freezing and thawing can therefore cause concrete to crack quickly. However, returning to the subject of solutions, it is worth noting the waterproofing properties of these polymers. The research of ethylene-vinyl acetate is an example. 


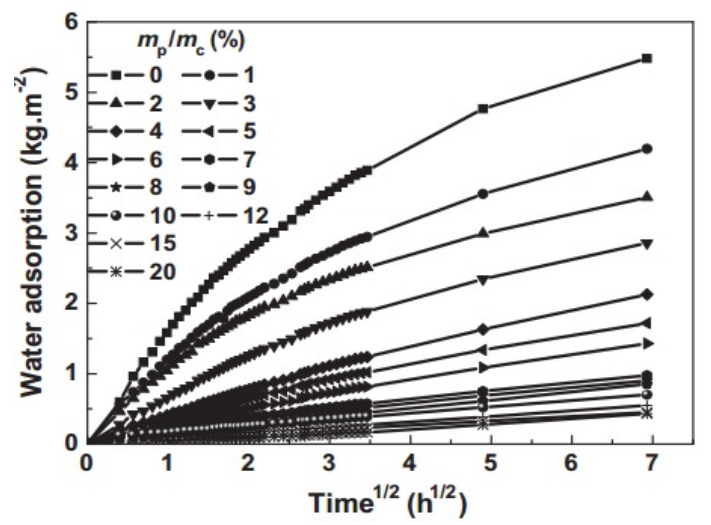

Fig.4 Relationship between the water capillary adsorption and time ( $m_{p}$-amount of polymer; $m_{c}$-amount of concrete) Source : [5]

As seen in Figure 4, the greatest impact on water resistance takes place at 7\%. Note that the biggest jump takes place between $1-7 \%$ then later between 7 and $20 \%$. This should be looked at from an economic perspective, as the more polymer in the mortar, the more expensive it will be. It is worth looking at the dependence at $20 \%$. At this amount of polymer content, e.g. SBS, water practically does not penetrate the cement.

A very interesting study was made by Schulze [6]. His task was to compare selected polymers and choose the best one. These were RE 545 (vinyl-acetate-ethylene powder), RI 551 Z (ethylene-vinylchlorid-vinyllaurate), LL 512 (styrene / acrylic powder). As for water impermeability, the best was RI 551 Z, which at $2 \%$ the absorption level was almost 0.

\section{8d NC 11 Year ODEX $\square 5$ Years ODEX}

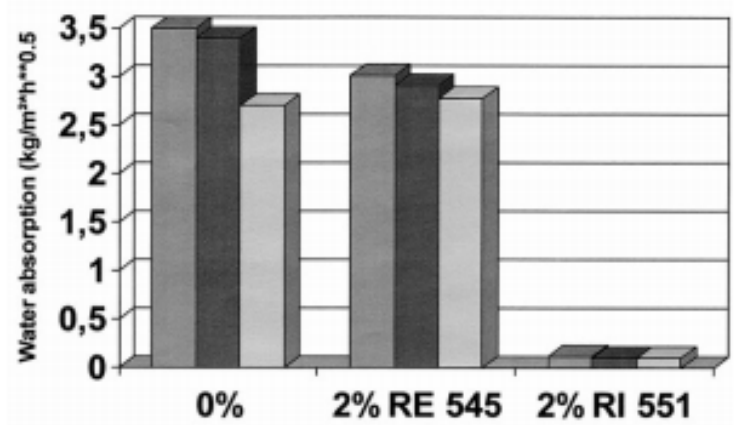

Fig. 5 Water absorption for non-modified cement and with RE 545 and RI 551 (NC-normal climate, ODEX-outdoor exposure, RE 545 -vinylacetate-ethylene powder, RI 551 -ethylene-vinylchlorid-vinyllaurate)

Source : [6]

As shown, RI 551 polymer allows to achieve an impermeable surface even at a low content of $2 \%$. It is a polymer which deserves further study as it gives very good results not only in the case of waterproofness but also flexural and compression strength. However, there is one disturbing factor using a polymer. Flexural strength in the case of external and internal samples for unmodified cement differs, because the same internal strength is obtained after 10 years whereas the external sample is obtained after 4 years. This is due to the low water content in internal conditions. Schulze's study [6] is important in the context of long-term research, as it allows to determine the decrease or increase in the value of given properties.

\section{Discussion and conclusions}

On the basis of the presented research it can be concluded that the addition of polymer during cement formation has advantages. It not only increases compressive strength, flexural strength and more importantly, water resistance, but also creates a more compressible surface providing new insulating properties. In the case of modern low energy buildings structures, such a material not only achieves the ability to retain heat but will also allow the use of less of other insulation materials, e.g. mineral wool. However, this solution also has several 
disadvantages. One is undoubtedly the price of polymer and the problems with mixing. Another important disadvantage is the length of time for external conditions to be established. With the use of an impermeable layer, compressive strength reaches its $100 \%$ capacity only after 10 years.

Nevertheless, there are many indications that cement reinforced with polymers will be widely used in the future. The conditions that must be met are primarily increased hardness, resistance to weather conditions but also the important economic aspect. First, the cement must be made more resistant to moisture, which causes corrosion of the steel inside the cement. The proper polymer must not only be efficient, but also chemically neutral. It is also important to determine the right amount of polymer to be added depending on the application. This especially concerns constructions where high strength is required, such as layers of concrete under supports and beams, as well as marine constructions or roof coverings most exposed to weather conditions. The main area of improvement or development of polymers may concern composites based on monomers. Hemp from the paper industry is also very promising. Another important issue is repair material in the form of various types of cement masses or pastes. In places where there has been damage such as where the cement is reinforced, repairs can be extremely difficult, because the corrosion process cannot be inhibited. Therefore, scientists should find a polymer that will not only fill the damaged section but also neutralize corrosion. In order to apply polymer technology scientists should strive to find an economically feasible solution.

\section{References}

[1] A. Sharma,V. Tyagi,C. Chen,D. Buddhi, Review on thermal energy storage with phase change materials and applications, Renewable and Sustainable Energy Reviews 13, (2007) 318-345

[2] P. Lukic, J. Tamburic, D. Stojić, Energy efficiency of buildings with phase-change materials, Architecture and Civil Engineering, (2012), $343-352$,

[3] D.W. Fowler, Polymers in concrete: a vision for the 21st century, Cement and concrete, (1999), 449-452

[4] R. Wang, P. Wang, X. Li, Physical and mechanical properties of styrene-butadiene rubber emulsion modified cement mortars, Cement and Concrete Research 35,(2004) 900-906,

[5] R. Wang, P. Wang, Action of redispersible vinyl acetate and versatate copolymer powder in cement mortar, Construction and Building Materials 25, (2011), 4210-4214,

[6] J. Schulze, O. Killermann, Long-term performance of redispersible powders in mortars, Cement and Concrete Research 31, (2000) 357-362,

[7] $\mathrm{ACl}$ Committee 548, Guide for the Use of Polymers in Concrete, (1994),

[8] F. Shaker, A. El-Dieb, M. Reda, Durability of styrene-butadiene latex modified concrete, Cement and Concrete Research 27,(1997) 711-720,

[9] S.M. Halliwell, Polymers in Building and Construction, Rapra review reports, (2003), volume 13,

[10] M. Frigione, Concrete with polymers, Woodhead Publishing Limited, (2013), 386-436, 